\title{
The evolution of gravitons in accelerating cosmologies: the case of extended gravity
}

\author{
S. Capozziello ${ }^{1,2,3}$, M. De Laurentis ${ }^{4,5,6,2}$, S. Nojiri ${ }^{7,8}$, S. D. Odintsov ${ }^{9,10}$ \\ 1 Dipartimento di Fisica "E. Pancini", Università di Napoli "Federico II" \\ ${ }^{2}$ INFN Sez. di Napoli, Compl. Univ. di Monte S. Angelo, Edificio G, Via Cinthia, I-80126, Napoli, Italy, \\ ${ }^{3}$ Gran Sasso Science Institute, Via F. Crispi 7, I-67100, L' Aquila, Italy, \\ ${ }^{4}$ Institute for Theoretical Physics, Goethe University, \\ Max-von-Laue-Str. 1, 60438 Frankfurt, Germany, \\ ${ }^{5}$ Tomsk State Pedagogical University, 634061 Tomsk, Russia \\ 6 Laboratory for Theoretical Cosmology, Tomsk State University of \\ Control Systems and Radioelectronics (TUSUR), 634050 Tomsk, Russia \\ 7 Department of Physics, Nagoya University, Nagoya 464-8602, Japan \\ 8 Kobayashi-Maskawa Institute for the Origin of Particles and the Universe, Nagoya University, Nagoya 464-8602, Japan \\ ${ }^{9}$ ICREA, Passeig Luis Companys, 23, 08010 Barcelona, Spain, \\ 10 Institute of Space Sciences (IEEC-CSIC) C. Can Magrans s/n, 08193 Barcelona, Spain
}

(Dated: April 14, 2017)

\begin{abstract}
We discuss the production and evolution of cosmological gravitons showing how the cosmological background affects their dynamics. Besides, the detection of cosmological gravitons could constitute an extremely important signature to discriminate among different cosmological models. Here we consider the cases of scalar-tensor gravity and $f(R)$ gravity where it is demonstrated the amplification of graviton amplitude changes if compared with General Relativity. Possible observational constraints are discussed.
\end{abstract}

PACS numbers: 04.30, 04.30.Nk, 04.50.+h, 98.70.Vc

\section{INTRODUCTION}

The recent discovery of gravitational waves [1, 2] opened the doors to the so called gravitational astronomy by which a new fundamental tool to explore the universe is at hand. Despite this amazing result, several open issues remain to be addressed in cosmology and astrophysics, first of all the puzzle of dark side. This issue could have a "material" explanation by finding out new particles beyond the Standard Model or be addressed by extending the General Relativity (GR) including further degrees of freedom like scalar fields or further geometric invariants related to curvature or torsion [3 12$]$.

Gravitational radiation could have a major role in this perspective because both the production and the evolution of gravitons could probe the universe at various scales [13]. Furthermore, the features of gravitational radiation could be an intrinsic way to test theories 14]. In fact, further gravitational modes related to the extensions of GR could be revealed both at astrophysical and cosmological scales [15 19], once suitable interferometric experiments will be available. To this end, in order to find out further polarizations of gravitational waves, more than two interferometric antennas are needed to disentangle other modes with respect to the standard $\times$ and + modes of GR [20].

The aim of this paper is to show that the production and the evolution of gravitons can trace the evolution of cosmological models and then, in principle, distinguish among competing gravitational theories.

Gravitons are an ideal tool because they do not interact with any form of matter and can be originated at the origin of the universe as vacuum fluctuations. Due to these characteristics, gravitons could be related to the whole cosmic history. In particular, the behavior of cosmic accelerating/decelerating background is intrinsically related to the shape of graviton amplitudes evolving with time.

The paper is structured as follows. Sec. II deals with the general problem of gravitons evolving in an expanding universe. Their conformal properties are discussed. Sec. III is devoted to the production of gravitons by inflationary mechanism. Such gravitons could constitute the main ingredient of the gravitational stochastic background. In Secs. IV and $\mathrm{V}$ scalar tensor and $f(R)$ gravity are considered respectively. The goal is to demonstrate that the behavior of gravitons can distinguish cosmological features (e.g. phantom phases [21] and Big Rip singularities [22]) and then discriminate among models. Discussion and conclusions are drawn in Sec. VI. Here we discuss also possible experimental constraints that could be considered to implement the present approach.

\section{GRAVITONS IN EXPANDING UNIVERSE AND THEIR CONFORMAL PROPERTIES}

Let us consider the equation of motion for gravitons in the expanding universe. The expansion of the universe is generated by the energy momentum tensor of matter acting as a source. However, the concept of source is very 
general and any (material and geometric) contribution to the right-hand side of the Einstein field equations can contribute. The metric dependence of the energy momentum tensor is not always straightforward and modifications can be induced by scalar fields, geometric invariants and any source contributing to the expansion of the universe (see for example Ref. [23]).

Before considering the cosmological applications, it is worth discussing conformal properties of graviton dynamics in a given expanding background. These considerations are worth in view of understanding how the evolution of gravitons can be used to probe different cosmological backgrounds. As we will show, the approach works for models where it is possible to disentangle the standard fluid matter with respect to the geometry into the Einstein field equations. In general, perturbing the metric means

$$
g_{\mu \nu} \rightarrow g_{\mu \nu}+h_{\mu \nu}
$$

where $\left|h_{\mu \nu}\right| \ll 1$ is the perturbation with respect to a given background $g_{\mu \nu}$. It is straightforward to obtain the perturbed Ricci tensor and scalar,

$$
\begin{aligned}
\delta R_{\mu \nu} & =\frac{1}{2}\left[\nabla_{\mu} \nabla^{\rho} h_{\nu \rho}+\nabla_{\nu} \nabla^{\rho} h_{\mu \rho}-\nabla^{2} h_{\mu \nu}-\nabla_{\mu} \nabla_{\nu}\left(g^{\rho \lambda} h_{\rho \lambda}\right)-2 R_{\nu}^{\lambda}{ }_{\mu} h_{\lambda \rho}+R_{\mu}^{\rho} h_{\mu \nu}+R_{\nu}^{\rho} h_{\rho \mu}\right], \\
\delta R & =-h_{\mu \nu} R^{\mu \nu}+\nabla^{\mu} \nabla^{\nu} h_{\mu \nu}-\nabla^{2}\left(g^{\mu \nu} h_{\mu \nu}\right) .
\end{aligned}
$$

We are not considering the perturbation of scalar fields because the spin two field, i.e. the graviton, does not mix with the scalar field (spin zero) field. In this sense, the "genuine" graviton is a tensor field propagating into a given background. However, it is worth noticing that extending GR means to take into account further degrees of freedom that can be figured out as effective scalar fields [11].

By imposing the gauge condition

$$
\nabla^{\mu} h_{\mu \nu}=g^{\mu \nu} h_{\mu \nu}=0
$$

the Einstein field equations

$$
R_{\mu \nu}-\frac{1}{2} g_{\mu \nu} R=\kappa^{2} T_{\mu \nu}
$$

assume the following perturbed form:

$$
\frac{1}{2}\left[-\nabla^{2} h_{\mu \nu}-2 R_{\nu}^{\lambda}{ }_{\nu}{ }_{\mu} h_{\lambda \rho}+R_{\mu}^{\rho} h_{\rho \nu}+R_{\nu}^{\rho} h_{\rho \mu}-h_{\mu \nu} R+g_{\mu \nu} R^{\rho \lambda} h_{\rho \lambda}\right]=\kappa^{2} \delta T_{\mu \nu} .
$$

Here we are assuming that the energy-momentum tensor perturbations, acting as a source, lead the evolution of the gravitons. This means that any source contributing to the r.h.s. of Eq. (6) affects the propagation of gravitons. However, before considering the form of $\delta T_{\mu \nu}$, we have to discuss conformal properties of gravitons showing how further degrees of freedom of gravitational field can be figured out as further scalar fields acting as sources.

In general, gravitational waves derive from perturbations $h_{\mu \nu}$ of the metric $g_{\mu \nu}$ and transform as 3-tensors. The gravitational wave equation in vacuum and in the transverse-traceless gauge is

$$
\square h_{i}{ }^{j}=0,
$$

where $\square$ is the d'Alembert operator defined as $\square \equiv(-g)^{-1 / 2} \partial_{\mu}(-g)^{1 / 2} g^{\mu \nu} \partial_{\nu}$. This equation comes directly from the Einstein field Eqs. (5) in the Minkowskian limit. The Latin indexes are for spatial coordinates and the Greek ones for spacetime coordinates. Our task is now to derive the analog of Eqs. (7) assuming a generic theory of gravity conformally related to GR.

Assuming the conformal transformation

$$
\widetilde{g}_{\mu \nu}=\mathrm{e}^{2 \Phi} g_{\mu \nu},
$$

where $\Phi$ is the conformal scalar field, the Ricci tensor and scalar are respectively

$$
\widetilde{R}_{\mu \nu}=R_{\mu \nu}+2\left(\Phi_{; \mu} \Phi_{; \nu}-g_{\mu \nu} \Phi_{; \delta} \Phi^{; \delta}-\Phi_{; \mu \nu}-\frac{1}{2} g_{\mu \nu} \Phi_{; \delta}^{; \delta}\right), \quad \widetilde{R}=\mathrm{e}^{-2 \Phi}\left(R-6 \square \Phi-6 \Phi_{; \delta} \Phi^{; \delta}\right),
$$

where the scalar part can be easily disentangled with respect to the tensor part.

The box operator transforms in the conformal metric as

$$
\widetilde{\square} \equiv(-\widetilde{g})^{-1 / 2} \partial_{\mu}(-\widetilde{g})^{1 / 2} \widetilde{g}^{\mu \nu} \partial_{\nu},
$$


and then, applied to Eq.(8), gives

$$
\widetilde{\square}=\mathrm{e}^{-2 \Phi}\left(\square+2 \Phi^{; \lambda} \partial_{; \lambda}\right)
$$

This means that the gravitational wave equation in the Jordan frame becomes

$$
\widetilde{\square} \widetilde{h}_{i}^{j}=0
$$

expressed in the conformal metric $\widetilde{g}_{\mu \nu}$.

Since no scalar perturbation couples to the tensor part, we can discard the $\delta \Phi$ contribution and, being $\widetilde{h}_{i}{ }^{j}=\widetilde{g}^{l j} \delta \widetilde{g}_{i l}=\mathrm{e}^{-2 \Phi} g^{l j} \mathrm{e}^{2 \Phi} \delta g_{i l}=h_{i}{ }^{j}$. In this sense, $h_{i}{ }^{j}$ results conformally invariant. The wave amplitude is then

$$
h(t)_{i}^{j}=h(t) e_{i}^{j} \exp \left(i k_{l} x^{l}\right)
$$

where $e_{i}^{j}$ is the polarization tensor for both metrics. Time evolution is given by the scalar amplitude function $h(t)$, where we are assuming that time and space coordinates are disentangled in the evolution of gravitons. The meaning of the formula (11) is that the background conformally changes. In other words, the gravitational waves can be used to test different cosmological backgrounds. Below, we will specify the conformal transformations for scalar tensor and $f(R)$ gravity for Friedmann-Robertson-Walker (FRW) universes. In other words, we will consider FRW universe in physical time and in conformal time description taking into account the evolution of gravitons. Finally, an important point has to be stressed. The characteristic properties of the gravitational waves are preserved under general conformal transformations where $\Phi$ is a general spacetime function. In particular, the transverse property is always preserved by a conformal transformation if $\widetilde{\nabla}^{\mu} \widetilde{h}_{\mu \nu}=0$.

Although $\widetilde{h}_{\mu}^{\nu}=h_{\mu}^{\nu}$, even if $\nabla^{\mu} h_{\mu}^{\nu}=0$, in general on finds $\widetilde{\nabla}^{\mu} \widetilde{h}_{\mu}^{\nu} \neq 0$. In fact we have,

$$
\widetilde{\nabla}^{\mu} \widetilde{h}_{\mu}^{\nu}=\mathrm{e}^{-\Phi} \nabla^{\mu} h_{\mu \nu}+D \mathrm{e}^{-\Phi} g^{\mu \sigma} g^{\nu \rho} \Phi_{, \sigma} h_{\mu \rho}-\mathrm{e}^{-\Phi} g^{\nu \rho} \Phi_{, \rho} g^{\mu \sigma} h_{\mu \sigma}
$$

where $D$ is the dimensions of space-time. Then even if we impose the gauge condition (44), we obtain

$$
\widetilde{\nabla}^{\mu} \widetilde{h}_{\mu}^{\nu}=D \mathrm{e}^{-\Phi} g^{\mu \sigma} g^{\nu \rho} \Phi_{, \sigma} h_{\mu \rho}
$$

If we assume that the background metric and therefore $\Phi$ only depend on the cosmological time $t$ and we consider the perturbation with $h_{t \mu}=0$, the r.h.s. in (15) vanishes, and then

$$
\widetilde{\nabla}^{\mu} \widetilde{h}_{\mu}^{\nu}=0
$$

Therefore the equation for the graviton is not changed by the conformal transformation (8). In this sense, as we will discuss below, gravitational waves, i.e. gravitons, can be an efficient tool to probe the cosmological background.

A first systematic study in this sense is reported in [24]. These authors studied the behavior of cosmological gravitational waves under conformal transformations pointing out that information carried in Einstein's and in Jordan's frame are different. This fact is extremely important in order to discriminate the physical frame.

\section{THE PRODUCTION OF COSMOLOGICAL GRAVITONS}

Before considering specific backgrounds, it is worth discussing the mechanism of production of cosmological gravitons. In general, the cosmic evolution of gravitons is strictly related to the problem of their production. It is worth stressing that cosmological gravitons contribute to the stochastic background and can be generated by several mechanisms of cosmological and astrophysical origin [26, 27]. As a general remark, the gravitational stochastic background is essentially due to very energetic phenomena in early universe and it is strictly related to the cosmological model. It is possible to show that the graviton evolution can be connected to given cosmological models assuming that contributions to the stochastic background come from the vacuum fluctuations originated at primordial inflation eras. The paradigm is that a transition occurs between a superluminal (e.g. exponential or power law phase) and a Friedmann power-law phase. Gravitons adiabatically evolve in relation to damped oscillations $(\sim 1 / a)$. Here $a$ is the FRW scale factor. The process stops when gravitons reach the Hubble radius $H^{-1}$. Here the Hubble size is the perturbation particle horizon. Further fluctuations are negligible thanks to the inflation. Gravitons freeze out at $a / k \gg H^{-1}$. As soon as the reheating starts, gravitons reenter the Hubble radius. Depending on the graviton scale perturbations, such a reenter can happen at radiation era or at dust era. The Sachs-Wolfe effect on the temperature anisotropy $\triangle T / T$ is the way to detect the phenomenon [28]. 
The mechanism of graviton production can be outlined as follows. Let us consider a scalar field $\Phi$ acting as the inflaton. In order to have inflation, it has to be $\dot{\Phi} \ll H$, where the dot represent the derivative with respect to cosmic time. It is worth defining a conformal time $d \sigma=d t / a$. Then the conformal gravitational wave equation (12) in FRW metric, adopting the definition (11), becomes

$$
h^{\prime \prime}+2\left(\frac{\chi^{\prime}}{\chi}\right) h^{\prime}+k^{2} h=0,
$$

where $\chi=a \mathrm{e}^{\Phi}$ and derivation is with respect to $\sigma$. Clearly, the given cosmological model is assigned by $\Phi$.

The mechanism of production can be realized as follows. The inflationary background is given, in general, by $a(t)=a_{0} \exp (H t)$ and then $\sigma=\int d t / a=(a H)^{-1}$. This means that, inside (17), it is $\chi^{\prime} / \chi=-\sigma^{-1}$. The solution of (17) is

$$
h(\sigma)=k^{-3 / 2} \sqrt{2 / k}\left[C_{1}(\sin k \sigma-\cos k \sigma)+C_{2}(\sin k \sigma+\cos k \sigma)\right],
$$

where $C_{1,2}$ are integration constants. We can distinguish between two regimes, inside and outside the Hubble radius. Inside the Hubble radius $H^{-1}$, it is $k \sigma \gg 1$. Supposing that the initial vacuum state is symmetric, this means that we have no initial graviton and then only negative-frequency modes are generated. The adiabatic behavior is then

$$
h=k^{1 / 2} \sqrt{2 / \pi}\left(\frac{1}{a H}\right) C \exp (-i k \sigma),
$$

with $C$ again a constant. The change of regime is realized at the first horizon crossing $(a H=k)$. Here, the averaged amplitude $A_{h}=(k / 2 \pi)^{3 / 2}|h|$ of the perturbation can be assumed as

$$
A_{h}=\frac{1}{2 \pi^{2}} C
$$

As soon as the scale $a / k$ becomes larger than $H^{-1}$, the growing modes are constant and result frozen. This situation is realized for $-k \sigma \ll 1 \mathrm{in} \mathrm{Eq.} \mathrm{(18).} \mathrm{The} \mathrm{inflaton} \mathrm{field} \mathrm{is} \Phi \sim 0$ at reenter. As a consequence the amplitude $A_{h}$ of the graviton remain the same up to the second horizon crossing. It can be observed as anisotropy perturbation on the microwave background. In particular $A_{h}$ is the upper limit on the cosmological temperature perturbation which is

$$
\triangle T / T \lesssim A_{h}
$$

and this means that other effects can bring contributions to the background anisotropy [29]. In Eq. (21), the important quantity is $C$, the amplitude in Eq. (19). It is conserved up to the reenter. It strictly depends on inflation that produce perturbations by means of zero-point energy fluctuations.

Specifically the production mechanism depends on the specific theory of gravity that gives the background and then the inflationary behavior. From an observational point of view, $(\triangle T / T)$, through $A_{h}$, is a further constraint to select cosmological models and then to select possible modified gravity theories [30].

Coming into details, one can explicitly show how the graviton amplitude is related to the background and the field sourcing the inflation. Let us take into account a single graviton described as a monochromatic wave. Its zero-point amplitude is derived from the commutation relation:

$$
\left[h(t, x), \pi_{h}(t, y)\right]=i \delta^{3}(x-y),
$$

calculated at time $t$. Here the amplitude $h$ has the role of the field and $\pi_{h}$ is its conjugate momentum. One can write an effective interaction Lagrangian for $h$ as

$$
\widetilde{\mathcal{L}}=\frac{1}{2} \sqrt{-\widetilde{g}} \widetilde{g}^{\mu \nu} h_{; \mu} h_{; \nu}
$$

in the conformal metric $\widetilde{g}_{\mu \nu}$. From this Lagrangian, the conjugate momentum is

$$
\pi_{h}=\frac{\partial \widetilde{\mathcal{L}}}{\partial \dot{h}}=\mathrm{e}^{2 \Phi} a^{3} \dot{h} .
$$

Immediately, the formal Eq. (22) becomes

$$
[h(t, x), \dot{h}(t, y)]=i \frac{\delta^{3}(x-y)}{a^{3} \mathrm{e}^{2 \Phi}} .
$$


Functions $h$ and $\dot{h}$ are expandable through creation and annihilation operators, i.e.

$$
h(t, x)=\frac{1}{(2 \pi)^{3 / 2}} \int d^{3} k\left[h(t) \mathrm{e}^{-i k x}+h^{*}(t) e^{+i k x}\right], \quad \dot{h}(t, x)=\frac{1}{(2 \pi)^{3 / 2}} \int d^{3} k\left[\dot{h}(t) \mathrm{e}^{-i k x}+\dot{h}^{*}(t) \mathrm{e}^{+i k x}\right] .
$$

Taking into account the conformal time, the commutation relations are

$$
\left[h h^{\prime *}-h^{*} h^{\prime}\right]=\frac{i(2 \pi)^{3}}{a^{3} \mathrm{e}^{2 \Phi}} .
$$

Considering the solution (19) and (20) of cosmological equation (17), we obtain $C=\sqrt{2} \pi^{2} H \mathrm{e}^{-\Phi}$ where $H$ and $\Phi$ are calculated at the first horizon-crossing. Explicitly we obtain

$$
A_{h}=\frac{\sqrt{2}}{2} H \mathrm{e}^{-\Phi}
$$

which means that the amplitude of gravitons produced during inflation directly depends on the cosmological model (see also [31, 32] for a detailed discussion on this point). Clearly, $\mathrm{e}^{\Phi}=1$ for GR. Below, we shall consider two relevant cases, scalar-tensor and $f(R)$ gravity, showing that graviton evolution can be a relevant tool to discriminate features of cosmological models.

\section{THE SCALAR TENSOR CASE}

Let us develop the above considerations for some specific theories of gravity. First of all, we have to specify the explicit form of $\delta T_{\mu \nu}$. Assuming the scalar field model in [33] whose action is given by

$$
S_{\phi}=\int d^{4} x \sqrt{-g} \mathcal{L}_{\phi}, \quad \mathcal{L}_{\phi}=-\frac{1}{2} \omega(\phi) \partial_{\mu} \phi \partial^{\mu} \phi-V(\phi),
$$

we find

$$
T_{\mu \nu}=-\omega(\phi) \partial_{\mu} \phi \partial_{\nu} \phi+g_{\mu \nu} \mathcal{L}_{\phi}
$$

and therefore

$$
\delta T_{\mu \nu}=h_{\mu \nu} \mathcal{L}_{\phi}+\frac{1}{2} g_{\mu \nu} \omega(\phi) \partial^{\rho} \phi \partial^{\lambda} \phi h_{\rho \lambda},
$$

up to first order in perturbations. We are interested in the evolution of tensor gravitons so we can consider only the spatial component of $h_{\mu \nu}$, that is $h_{i j}$.

Assuming a FRW spatially flat metric

$$
d s^{2}=-d t^{2}+a(t)^{2} \sum_{i=1,2,3}\left(d x^{i}\right)^{2}
$$

and $\phi=t$ in (31), the FRW equations are

$$
\frac{3}{\kappa^{2}} \frac{\dot{a}^{2}}{a^{2}}=\frac{\omega}{2}+V, \quad-\frac{1}{\kappa^{2}}\left(2 \frac{\ddot{a}}{a}+\frac{\dot{a}^{2}}{a^{2}}\right)=\frac{\omega}{2}-V .
$$

We find

$$
\omega=-\frac{2}{\kappa^{2}}\left(\frac{\ddot{a}}{a}-\frac{\dot{a}^{2}}{a^{2}}\right), \quad V=\frac{1}{\kappa^{2}}\left(\frac{\ddot{a}}{a}+2 \frac{\dot{a}^{2}}{a^{2}}\right),
$$

for the kinetic term and the scalar field potential expressed as functions of the scale factor $a(t)$ and its derivatives. By using (6), (31), (34), and $\phi=t$, we find the evolution equation of graviton:

$$
0=\left(2 \frac{\ddot{a}}{a}+4 \frac{\dot{a}^{2}}{a^{2}}+\frac{\dot{a}}{a} \partial_{t}-\partial_{t}^{2}+\frac{\triangle}{a^{2}}\right) h_{i j},
$$


which clearly depends on the cosmological background. We have to compare Eq. (35) with the expression in case of $\delta T_{\mu \nu}=0$. We obtain

$$
0=\left(6 \frac{\ddot{a}}{a}+6 \frac{\dot{a}^{2}}{a^{2}}+\frac{\dot{a}}{a} \partial_{t}-\partial_{t}^{2}+\frac{\triangle}{a^{2}}\right) h_{i j},
$$

where the different coefficients specify the role of scalar field $\phi$, and then the matter source, in the evolution. Clearly, Eqs. (35) and (36) are conformally related according to the discussion in Sec. II].

Let us now investigate the solution of Eq. (35). By rewriting Eq. (35) as follows,

$$
0=-a^{3} \partial_{t}\left(a^{3} \partial_{t}\left(a^{-2} h_{i j}\right)\right)+4 a^{4} \dot{a}^{2}\left(a^{-2} h_{i j}\right)+a^{4} \triangle\left(a^{-2} h_{i j}\right),
$$

and assuming $h_{i j}$ given by the above spatially plane wave (13), one has

$$
h_{i j}(\boldsymbol{x}, t)=\mathrm{e}^{i \boldsymbol{k} \cdot \boldsymbol{x}} a(t)^{2} \hat{h}_{i j}(t) .
$$

We can define a new conformal time coordinate $\tau$ as $^{1}$

$$
d \tau=a(t)^{-3} d t
$$

and then we obtain

$$
0=\frac{d^{2} \hat{h}_{i j}}{d \tau^{2}}+4 a^{4} \dot{a}^{2} \hat{h}_{i j}+k^{2} a^{4} \hat{h}_{i j} .
$$

Here $k^{2} \equiv \boldsymbol{k} \cdot \boldsymbol{k}$ is the wave number of Eq. (17).

To study the evolution of gravitons after the inflationary production, let us consider a power-law behavior for the scale factor of the universe, that is

$$
a(t)=\left(\frac{t}{t_{0}}\right)^{\alpha}
$$

with $t_{0}$ and $\alpha$ real constants. Depending on the value of $\alpha$, Eq. (41) involves a power law (superluminal) inflation $(\alpha \geq 1)$, a Friedmannian (subluminal) evolution $(0<\alpha<1)$, and a pole-like (phantom) behavior $(\alpha<0)$.

The universe given by (41) can be realized by the scalar-tensor model in (29) by substituting (41) into the expressions for $\omega(\phi)$ and $V(\phi)$ given in (34), that is

$$
\omega(\phi)=\frac{2 \alpha}{\kappa^{2} t_{0}^{2} \phi^{2}}, \quad V(\phi)=\frac{3 \alpha^{2}-\alpha}{\kappa^{2} t_{0}^{2} \phi^{2}} .
$$

Eq. (39) tells us that, if $\alpha \neq \frac{1}{3}$, the conformal time is

$$
\tau=\frac{t_{0}}{1-3 \alpha}\left(\frac{t}{t_{0}}\right)^{1-3 \alpha}
$$

Therefore $t \rightarrow 0$ corresponds to $\tau \rightarrow 0$ if $\alpha<\frac{1}{3}$ and $\tau \rightarrow-\infty$ if $\alpha>\frac{1}{3}$. On the other hand $t \rightarrow+\infty$ corresponds to $\tau \rightarrow+\infty$ if $\alpha<\frac{1}{3}$ and $\tau \rightarrow 0$ if $\alpha>\frac{1}{3}$. It is worth noticing that $\alpha>\frac{1}{3}$ corresponds to the accelerating expansion of the universe and $\alpha<\frac{1}{3}$ to the decelerating expansion. From a physical point of view, a(t) $\sim t^{1 / 3}$ represents a stiff-matter behavior [25]. Furthermore we have that

$$
k^{2} a^{4}=k^{2}\left(\frac{\tau}{\tau_{0}}\right)^{\frac{4 \alpha}{1-3 \alpha}}, \quad 4 a^{4} \dot{a}^{2}=\frac{4 \alpha^{2}}{t_{0}^{2}}\left(\frac{\tau}{\tau_{0}}\right)^{-2} .
$$

Here $\tau_{0} \equiv \frac{t_{0}}{1-3 \alpha}$. Because $\frac{4 \alpha}{1-3 \alpha}-(-2)=\frac{2(1-\alpha)}{1-3 \alpha}$, if $\alpha<\frac{1}{3}$ or $\alpha>1$, the first term in (44) dominates when $|\tau| \rightarrow \infty$ and the second term dominates when $\tau \rightarrow 0$. On the other hand, if $\frac{1}{3}<\alpha<1$, the second term in (44) dominates

\footnotetext{
${ }^{1}$ It is worth noticing that the conformal time $\sigma$ adopted above is useful for the scaling properties of the graviton while $\tau$ is considering the volume invariance related to $a^{3}$. The time $\tau$ can be always related to $\sigma$.
} 
when $|\tau| \rightarrow \infty$ and the first term dominates when $\tau \rightarrow 0$. In other words, the graviton behavior can discriminate among the various cosmological regimes. Let us now assume $\hat{h}_{i j}$ behaves asymptotically as

$$
\hat{h}_{i j} \propto \mathrm{e}^{\beta\left(\frac{\tau}{\tau_{0}}\right)^{\gamma}}
$$

Then

$$
\frac{d^{2} \hat{h}_{i j}}{d \tau^{2}}=\frac{1}{\tau_{0}^{2}}\left[\beta \gamma(\gamma-1)\left(\frac{\tau}{\tau_{0}}\right)^{\gamma-2}+\beta^{2} \gamma^{2}\left(\frac{\tau}{\tau_{0}}\right)^{2(\gamma-1)}\right] \hat{h}_{i j}
$$

Because $\gamma-2-2(\gamma-1)=-\gamma$, if $\gamma>0$, the first term in the r.h.s. of (46) dominates when $\tau \rightarrow 0$ and the second term dominates when $|\tau| \rightarrow \infty$. If $\gamma<0$, the second term in (46) dominates when $\tau \rightarrow 0$ and the first term dominates when $|\tau| \rightarrow \infty$.

When the first term in the r.h.s. of (46) dominate, by using (40) and (44), we find

$$
\gamma=\frac{2(1-\alpha)}{1-3 \alpha}, \quad \beta=-\frac{(1-3 \alpha)^{2} k^{2}}{2(1-\alpha)(1+\alpha)} .
$$

Then combining (43), (45), and (47), we find

$$
\hat{h}_{i j} \propto \mathrm{e}^{-\frac{(1-3 \alpha)^{2} k^{2}}{2(1-\alpha)(1+\alpha)}\left(\frac{t}{t_{0}}\right)^{2(1-\alpha)}}
$$

which is not a plane wave.

On the other hand, when the second term in (46) dominates, we find

$$
\gamma=\frac{1-\alpha}{1-3 \alpha}, \quad \beta= \pm i\left(\frac{1-3 \alpha}{1-\alpha}\right) k
$$

which corresponding to the oscillating mode. By using (43) and (45), and (49), we obtain

$$
\hat{h}_{i j} \propto \mathrm{e}^{ \pm i\left(\frac{1-3 \alpha}{1-\alpha}\right) k\left(\frac{t}{t_{0}}\right)^{1-\alpha}}
$$

The obtained expression is oscillating but it is not a simple plane wave except in the limit of $\alpha \rightarrow 0$. In the above two cases, we find that $\gamma>0$ when $0<\alpha<\frac{1}{3}$ or $\alpha>1$ and $\gamma<0$ when $\frac{1}{3}<\alpha<1$.

Furthermore when the second term (44) and the first term in (46) dominate, if we assume (51), we find $\gamma=0$, that is, $\hat{h}_{i j}$ becomes a constant, which may tell that we need an ansatz different from (45) like

$$
\hat{h}_{i j} \propto\left(\frac{\tau}{\tau_{0}}\right)^{\eta}
$$

with a constant $\eta$ and we find

$$
0=\frac{\eta(\eta-1)}{\tau_{0}^{2}}+\frac{4 \alpha^{2}}{t_{0}^{2}}
$$

that is

$$
\eta=\frac{1 \pm \sqrt{1-16 \alpha^{2} \frac{\tau_{0}^{2}}{t_{0}^{2}}}}{2}=\frac{1 \pm \sqrt{1-\frac{16 \alpha^{2}}{(1-3 \alpha)^{2}}}}{2} .
$$

In terms of the cosmic time $t, \mathrm{Eq}$. (51) can be written as

$$
\hat{h}_{i j} \sim\left(\frac{t}{t_{0}}\right)^{\eta(1-3 \alpha)} .
$$

In case that the second terms in (44) and (46) dominate, if we assume the behavior (51), we find $\gamma=0$, again, which tells us that $\hat{h}_{i j}$ is given by (51) and we re-obtain (52) and (53). It is worth noticing that $\eta$ becomes imaginary if $\alpha<-1$ or $\alpha>\frac{1}{7}$ but the real part of $\eta$ is always positive and, therefore, we find that when $\tau \rightarrow 0, \hat{h}_{i j} \rightarrow 0$ and when $|\tau| \rightarrow \infty, \hat{h}_{i j}$ diverges.

We can summarize the above considerations as follows. 
1. When $t \rightarrow 0$,

(a) $\alpha>1$. In this case, $t \rightarrow 0$ corresponds to $\tau \rightarrow-\infty$ as written after Eq. (43). Then the first term in (44) dominates. Furthermore because $\alpha>1$ tells $\gamma>0$, the second term in the r.h.s. of (46) dominates and therefore the solution oscillates but the absolute value of $\hat{h}_{i j}$ is finite.

(b) $\frac{1}{3}<\alpha<1$. Even in this case, $t \rightarrow 0$ corresponds to $\tau \rightarrow-\infty$. Because the second term in (44) dominates, the solution is given by (51) and diverges as in (54).

(c) $0<\alpha<\frac{1}{3}$ case. Because $t \rightarrow 0$ corresponds to $\tau \rightarrow 0$, the first term in (44) dominates and $\gamma>0$, which tells that the first term in the r.h.s. of (46) dominates and by using Eq. (48), we find $\hat{h}_{i j}$ goes to a finite value.

2. When $t \rightarrow+\infty$,

(a) $\alpha>1$. Because $t \rightarrow+\infty$ corresponds to $\tau \rightarrow 0$, the second term in (44) dominates and the solution is given by (54), which decreases for large $t$.

(b) $\frac{1}{3}<\alpha<1$. Because $t \rightarrow+\infty$ corresponds to $\tau \rightarrow 0$, again, the first term in (44) dominates. Then the second term in the r.h.s. of (46) dominates, the solution oscillates as in (50) and the absolute value of $\hat{h}_{i j}$ is finite.

(c) $0<\alpha<\frac{1}{3}$ case. Because $\gamma>0$ and $t \rightarrow+\infty$ corresponds to $\tau \rightarrow+\infty$, the solution is given by (50). Therefore the solution begins to oscillate but the absolute value of $\hat{h}_{i j}$ can be finite.

In summary, we find the enhacement of the gravitational wave when $\alpha>0$ in late time. Such a behavior could be interesting from an observational point of view. Because $\hat{h}_{i j}$ could be always finite, Eq. (38) tells that the gravitational wave $h_{i j}$ grows up by the factor $a(t)^{2}$.

For comparison, we can consider the model with $\delta T_{\mu \nu}=0$ in (36), that is when the scalar field does not act as a source for the gravitational equations. Then by using (38) and (39), instead of Eq. (40), we obtain

$$
0=\frac{d^{2} \hat{h}_{i j}}{d \tau^{2}}+\left(6 a^{4} \dot{a}^{2}+4 a^{6} \ddot{a}\right) \hat{h}_{i j}+k^{4} a^{4} \hat{h}_{i j} .
$$

In the case of the power-law scale factor in (41), instead of (44), we find

$$
k^{2} a^{4}=k^{2}\left(\frac{\tau}{\tau_{0}}\right)^{\frac{4 \alpha}{1-3 \alpha}}, \quad 6 a^{4} \dot{a}^{2}+4 a^{6} \ddot{a}=\frac{10 \alpha^{2}-4 \alpha}{t_{0}^{2}}\left(\frac{\tau}{\tau_{0}}\right)^{-2}
$$

Then as in (44), if $\alpha<\frac{1}{3}$ or $\alpha>1$, the first term in (44) dominates when $|\tau| \rightarrow \infty$ and the second term dominates when $\tau \rightarrow 0$. And furthermore, if $\frac{1}{3}<\alpha<1$, the second term in (44) dominates when $|\tau| \rightarrow \infty$ and the first term dominates when $\tau \rightarrow 0$. The first term in (56) is not changed from the first term in (44), there are some modifications if $\alpha<\frac{1}{3}$ or $\alpha>1$ and $\tau \rightarrow 0$, or if $\frac{1}{3}<\alpha<1$ and $|\tau| \rightarrow \infty$. In these cases, Eq. (52) is changed as

$$
0=\frac{\eta(\eta-1)}{\tau_{0}^{2}}+\frac{10 \alpha^{2}-4 \alpha}{t_{0}^{2}}
$$

and therefore we obtain

$$
\eta=\eta_{ \pm} \equiv \frac{1 \pm \sqrt{1-4\left(10 \alpha^{2}-4 \alpha\right) \frac{\tau_{0}^{2}}{t_{0}^{2}}}}{2}=\frac{1 \pm \sqrt{1-4 \frac{10 \alpha^{2}-4 \alpha}{(1-3 \alpha)^{2}}}}{2}
$$

In this case, the real part of $\eta$ is not always positive but if $0<\alpha<\frac{2}{5}, \eta_{-}$is negative. In conclusions, we can say that the cosmological evolution of the graviton depends on the cosmic background. Furthermore, the behavior of the graviton could be used to discriminate between the presence or not of a scalar field acting as a source in the field equations. 


\section{THE $f(R)$ CASE}

Similar considerations can be developed also in the case of $f(R)$ since the further degrees of freedom coming from the extension of GR (i.e. $f(R) \neq R$ ) can be always figured out as a further scalar field [3, 4]. For the sake of simplicity, let us assume a power-law $f(R)$ function of the form

$$
f(R) \sim R^{m}
$$

and a power-law scale factor (41). The exponents $\alpha$ and $m$ are related as follows

$$
\alpha=-\frac{(m-1)(2 m-1)}{m-2} .
$$

We have to note that there are extrema for $\alpha=-5 \pm 2 \sqrt{6}$ at $m=2 \mp \sqrt{\frac{3}{2}}$. In $f(R)$ gravity, the above conformal transformation (8) is

$$
g_{\mu \nu}=\frac{1}{f^{\prime}(R)} g_{\mathrm{E} \mu \nu}, \quad \text { being } \quad f^{\prime}(R)=\mathrm{e}^{2 \Phi},
$$

mapping the Jordan frame into to the Einstein frame. In the Einstein frame, the graviton modes and the scalar mode can be separated and we can easily find the equation of graviton (35). In the Einstein frame, the further degrees of freedom of $f(R)$ gravity can be recast as a scalar source in $T_{\mu \nu}$ and separated with respect to the evolution of the tensorial graviton. Below we will discuss the relative contributions of tensor and scalar modes in the stochastic background.

Considering again the power-law scale factor (41), the scalar curvature is proportional to $\frac{1}{t^{2}}$, then $f^{\prime}(R)$ can be written as

$$
f^{\prime}(R) \sim f_{0}\left(\frac{t}{t_{0}}\right)^{-2(m-1)}
$$

The metric (61) in the Einstein frame is given by

$$
d s_{E}^{2}=f_{0}\left(\frac{t}{t_{0}}\right)^{-2(m-1)}\left[-d t^{2}+\left(\frac{t}{t_{0}}\right)^{-\frac{2(m-1)(2 m-1)}{m-2}} \sum_{i=1,2,3}\left(d x^{i}\right)^{2}\right] .
$$

A time coordinate $t_{E}$ in the Einstein frame can be defined as follows

$$
t_{E}=t_{E 0}\left(\frac{t}{t_{0}}\right)^{2-m}
$$

Here

$$
t_{E 0} \equiv \frac{\sqrt{f_{0} t_{0}}}{2-m}
$$

It is worth noticing that $t \rightarrow 0$ corresponds to $t_{E} \rightarrow 0$, if $m<2$ but to $t_{E} \rightarrow \infty$, if $m>2$ and $t \rightarrow \infty$ corresponds to $t_{E} \rightarrow \infty$, if $m<2$ but to $t_{E} \rightarrow 0$ if $m>2$. Then the metric in the Einstein frame is given by

$$
d s_{E}^{2}=-d t_{E}^{2}+\left(\frac{t_{E}}{t_{E 0}}\right)^{6 \frac{(m-1)^{2}}{(m-2)^{2}}} \sum_{i=1,2,3}\left(d x^{i}\right)^{2} .
$$

Then $\tilde{\alpha} \equiv 3 \frac{(m-1)^{2}}{(m-2)^{2}}$ can be identified with $\alpha$ in (41) in the Einstein frame, which is different from $\alpha$ in the Jordan frame.

Since, in the Einstein frame, we can write the action in the scalar-tensor form, we can use the previous arguments. We find the following correspondence: 1) $\tilde{\alpha}=0 \Leftrightarrow \alpha=0,(m=1) ; 2) \tilde{\alpha}=+\infty \Leftrightarrow \alpha= \pm \infty,(m=2) ; 3)$ $\tilde{\alpha}=\frac{1}{3} \Leftrightarrow \alpha=0,\left(m=\frac{1}{2}\right)$ or $\left.\alpha=1,\left(m=\frac{5}{4}\right) ; 4\right) \tilde{\alpha}=1 \Leftrightarrow \alpha=1,\left(m=\frac{1 \pm \sqrt{3}}{2}\right)$. It is important to stress that there is no one-to-one correspondence between $\tilde{\alpha}$ and $m$ or $\alpha$. Then by using the results about the scalar tensor theory, we find: 
1. When $t \rightarrow 0$,

(a) $m<\frac{1-\sqrt{3}}{2}(\alpha>1,1<\tilde{\alpha}<3)$ : The solution $\hat{h}_{i j}$ oscillates but its absolute value is finite.

(b) $\frac{1-\sqrt{3}}{2}<m<\frac{1}{2}\left(0<\alpha<1, \frac{1}{3}<\tilde{\alpha}<1\right)$ : The solution is given by (51) and diverges as (54).

(c) $\frac{1}{2}<m<\frac{5}{4}\left(-5+2 \sqrt{6} \leq \alpha<\frac{1}{3}, 0<\tilde{\alpha}<\frac{1}{3}\right)$ : The solution $\hat{h}_{i j}$ goes to a finite value.

(d) $\frac{5}{4}<m<\frac{1+\sqrt{3}}{2}\left(\frac{1}{3}<\alpha<1, \frac{1}{3}<\tilde{\alpha}<1\right)$ : The solution is given by (511) and diverges as (54).

(e) $\frac{1+\sqrt{3}}{2}<m<2(\alpha>1, \tilde{\alpha}>1)$ : The solution $\hat{h}_{i j}$ oscillates but its absolute value is finite.

(f) $m>2(\alpha \leq-5-2 \sqrt{6}, \tilde{\alpha}>3)$ : We note that this case corresponds to $t_{E} \rightarrow \infty$. Then the solution decreases for small $t$.

2. When $t \rightarrow+\infty$,

(a) $m<\frac{1-\sqrt{3}}{2}(\alpha>1,1<\tilde{\alpha}<3)$ : The solution decreases for large $t$.

(b) $\frac{1-\sqrt{3}}{2}<m<\frac{1}{2}\left(0<\alpha<1, \frac{1}{3}<\tilde{\alpha}<1\right)$ : The solution oscillates and the absolute value of $\hat{h}_{i j}$ is finite.

(c) $\frac{1}{2}<m<\frac{5}{4}\left(-5+2 \sqrt{6} \leq \alpha<\frac{1}{3}, 0<\tilde{\alpha}<\frac{1}{3}\right)$ : The solution oscillates and the absolute value of $\hat{h}_{i j}$ is finite.

(d) $\frac{5}{4}<m<\frac{1+\sqrt{3}}{2}\left(\frac{1}{3}<\alpha<1, \frac{1}{3}<\tilde{\alpha}<1\right)$ : The solution oscillates and the absolute value of $\hat{h}_{i j}$ is finite.

(e) $\frac{1+\sqrt{3}}{2}<m<2(\alpha>1, \tilde{\alpha}>1)$ : The solution decreases for large $t$.

(f) $m>2(\alpha \leq-5-2 \sqrt{6}, \tilde{\alpha}>3)$ : This case corresponds to $t_{E} \rightarrow 0$ case. Therefore the solution oscillates but the absolute value of $\hat{h}_{i j}$ is finite.

Then we find that when $m<\frac{1-\sqrt{3}}{2}$ or $2>m>\frac{1+\sqrt{3}}{2}$, the amplitude of the solution is suppressed in the late time. For $m>2$ or $\frac{1}{2}<m<1, \alpha$ assumes negative values and therefore the universe undergoes a phantom evolution. It is worth noticing that the phantom universe does not appear in the case of scalar tensor theory. For phantom universe, $t \rightarrow \infty$ corresponds to the infinite past by changing the direction of time and $t \rightarrow 0$ corresponds to the Big Rip singularity. Near the Big Rip singularity, in case $m>2, \hat{h}_{i j}$ decreases; for $\frac{1}{2}<m<1, \hat{h}_{i j}$ is finite and not oscillating near the Big Rip singularity for $t \rightarrow 0$.

In the case $\alpha>0$, corresponding to the quintessence universe, $\hat{h}_{i j}$ decreases for both scalar-tensor and $f(R)$ gravity. We have to note that there is a difference in the exponent. In the case of scalar-tensor gravity, the exponent is given by (54) but in case of $f(R)$ gravity, by using (64), it is

$$
\hat{h}_{i j} \sim\left(\frac{t_{E}}{t_{E 0}}\right)^{\tilde{\eta}(1-3 \tilde{\alpha})}=\left(\frac{t}{t_{0}}\right)^{\tilde{\eta}(1-3 \tilde{\alpha})(-m+2)},
$$

which is the relation of the solution in the Einstein and in the Jordan frame.

Here $\tilde{\eta}$, instead of (53), is given by

$$
\tilde{\eta}=\tilde{\eta}_{ \pm} \equiv \frac{1 \pm \sqrt{1-\frac{16 \tilde{\alpha}^{2}}{(1-3 \tilde{\alpha})^{2}}}}{2} .
$$

We should also note that $\tilde{\alpha}$ is defined according to (66). For example, when $m \rightarrow-\infty$, we find $\alpha \rightarrow-m \rightarrow+\infty$ and $\tilde{\alpha} \rightarrow 3$ and therefore $\eta_{ \pm} \rightarrow \frac{1}{2} \pm \frac{i}{6}$ and $\tilde{\eta} \rightarrow \frac{1}{2} \pm i \frac{\sqrt{5}}{4}$. Then the exponent is given by

$$
\tilde{\eta}(1-3 \tilde{\alpha})(-m+2) \rightarrow\left(\frac{1}{2} \pm i \frac{\sqrt{5}}{4}\right)(-8)(-m)=-(4 \pm 2 i \sqrt{5}) \alpha .
$$

The corresponding exponent in (53), for the scalar tensor gravity, is

$$
\eta(1-3 \alpha) \sim-\left(\frac{3}{2} \pm i \frac{\sqrt{7}}{2}\right) \alpha .
$$

Then the decreasing exponent in the $f(R)$ gravity is much larger than the corresponding exponent in the scalar-tensor theory, the ratio is $8 / 3$. This means that if one is capable of fixing the exponent by observations, we can distinguish 
the $f(R)$ gravity from the scalar-tensor theory. In this way, the evolution of the gravitons can be a tool to discriminate among competing cosmological models.

We stress again that, although the phantom phase does not appear in the scalar-tensor theory, the phantom universe appears in $f(R)$ gravity and in the cases $\frac{1}{2}<m<1$ and $\alpha<0, \hat{h}_{i j}$ is finite even near the Big Rip singularity. Therefore we can easily distinguish the phantom universe from the quintessence phase with $\alpha>1$ in the scalar-tensor theory and the $f(R)$ gravity because $\hat{h}_{i j}$ decreases in the quintessence phase.

Some remarks are needed also regarding the Equation of State (EoS). Because the FRW equations in the Einstein gravity coupled with perfect fluid are given by,

$$
\rho_{\text {matter }}=\frac{3}{\kappa^{2}} H^{2}, \quad p_{\text {matter }}=-\frac{1}{\kappa^{2}}\left(3 H^{2}+2 \dot{H}\right)
$$

it is convenient to define the effective EoS parameter in terms of the Hubble rate $H \equiv \frac{\dot{a}}{a}$ as follows,

$$
w_{\mathrm{eff}}=-1-\frac{2 \dot{H}}{3 H^{2}} .
$$

For the power law expanding universe in (41), one finds $H=\frac{\alpha}{t}$ and therefore

$$
w_{\text {eff }}=-1+\frac{2}{3 \alpha} \text {. }
$$

Then the limit of $\alpha \rightarrow+\infty$ in (69) and (70) corresponds to the limit of $w_{\text {eff }} \rightarrow-1$, which is consistent with the $\Lambda$ CDM model. We have also to note that there is no one-to-one correspondence between $\alpha$ and $m$ in the $f(R)$ gravity as in (60). Besides, the limits $m \rightarrow-1$ and $m \rightarrow 2-0$ give $\alpha \rightarrow+\infty$. In the limit of $m \rightarrow 2-0$, we obtain the expression (70) of the scalar-tensor theory even for the $f(R)$ gravity and therefore it becomes difficult to distinguish $f(R)$ from scalar-tensor gravity.

In the scalar-tensor theory, there is an enhancement by the factor $a(t)^{2}$ because the effective gravitational wave $h_{i j}$ is related to $\hat{h}_{i j}$ by Eq. (38). Let us now investigate what happens in $f(R)$ gravity. In the Einstein frame, we have a relation similar to Eq. (38),

$$
h_{E i j}\left(\boldsymbol{x}, t_{E}\right)=\mathrm{e}^{i \boldsymbol{k} \cdot \boldsymbol{x}} a_{E}\left(t_{E}\right)^{2} \hat{h}_{E i j}\left(t_{E}\right) .
$$

The scale factor $a_{E}$ in the Einstein frame is related with scale factor $a$ in the Jordan frame by $a_{E}^{2}=f^{\prime}(R) a^{2}$ as given by (61). Furthermore Eq. (61) tells us that the gravitational wave $h_{i j}$ in the Jordan frame is related with the gravitational wave $h_{E i j}$ by $h_{i j}=f^{\prime}(R)^{-1} h_{E, i j}$, then we find

$$
h_{i j}(\boldsymbol{x}, t)=\mathrm{e}^{i \boldsymbol{k} \cdot \boldsymbol{x}} a(t)^{2} \hat{h}_{E i j}\left(t_{E}(t)\right) .
$$

Therefore there is an enhancement by a factor $a(t)^{2}$ but the situation is not changed with respect to that in the scalar tensor theory given in (61). As stated above, it is clear that the plane-wave amplitude evolution of the tensor graviton strictly depends on the background.

\section{DISCUSSON AND CONCLUSIONS}

As we said, the only realistic approach to investigate the above interplay between the cosmological background and the graviton evolution is the study of the stochastic background of gravitational waves. The stochastic background coming from gravitons can be mainly related to two phenomena: the (incoherent) superposition of gravitational waves generated by populations of astrophysical objects that cannot be resolved [34], and the primordial background of gravitational waves coming from early epochs [17, 35]. The stochastic background in described and characterized by a dimensionless spectrum [27, 36 38], that is

$$
\Omega_{\mathrm{GW}}^{A}(\nu)=\frac{1}{\rho_{c}}\left(\frac{d \rho_{\mathrm{GW}}^{A}}{d \ln \nu}\right), \quad \text { with } \quad \rho_{c} \equiv \frac{3 H_{0}^{2}}{8 \pi G}
$$


Here $\Omega_{\mathrm{GW}}^{A}(\nu)$ is an dimensionless density parameter, $\rho_{c}$ is the today cosmic critical energy density, $H_{0}$ the today observed Hubble parameter, and $d \rho_{\mathrm{GW}}$ is the energy density of the gravitational radiation in the frequency range $\nu$ to $\nu+d \nu$.

$$
\rho_{\mathrm{GW}}=\int_{0}^{\infty} d \nu \tilde{\rho}_{\mathrm{GW}}(\nu) .
$$

$\tilde{\rho}_{\mathrm{GW}}$ is the energy density of the gravitational waves per unit frequency. The relation between $\Omega_{\mathrm{GW}}(\nu)$ and the spectrum $S_{h}(\nu)$ is [27, 43]

$$
\Omega_{\mathrm{GW}}^{A}(\nu)=\left(\frac{4 \pi^{2}}{3 H_{0}^{2}}\right) f^{3} S_{h}^{A}(\nu)
$$

The energy density can be represented as $h_{0}^{2} \Omega_{\mathrm{GW}}(\nu)$ by assuming the dimensionless Hubble constant $H_{0}=$ $100 h_{0} \mathrm{~km} \mathrm{~s}^{-1} \mathrm{Mpc}^{-1}$. The stochastic background energy density is then written as

$$
\Omega_{\mathrm{GW}}^{A} \equiv \Omega_{\mathrm{GW}}^{+}+\Omega_{\mathrm{GW}}^{\times}+\Omega_{\mathrm{GW}}^{S},
$$

with $A=+, \times, S$. The first two terms come directly from GR while the third term comes from alternative gravity and is due to the scalar modes. In the case of $f(R)$ gravity, a scalar field can be achieved from $f^{\prime}(R) \rightarrow \Phi[44]$.

The stochastic background of gravitational waves is derived by the quantum fluctuations of zero-point energy. Such fluctuations are amplified in the early universe by the large variations of gravity. As discussed in Sec. III] this mechanism produces gravitational waves. The mechanism is in agreement with PLANCK data as well as with almost exponential inflation and spectral index $\approx 1,[30]$.

It is worth noticing that the inflationary scenario is related to a natural mechanism giving rise to perturbations for any field. Such a mechanism provides a spectrum for relic scalar gravitons which could constitute an important testbed for alternative gravity [36, 38 [41]. Introducing

$$
H_{\mathrm{ds}}^{2}=\frac{8 \pi G \rho_{\mathrm{ds}}}{3 c^{2}}, \quad \rho_{\text {Planck }}=\frac{c^{7}}{\hbar G^{2}},
$$

that is, the density at de Sitter (inflationary) epoch and the Planck density, respectively, the spectrum is given by

$$
\Omega_{\mathrm{GW}}^{A}(\nu)=\frac{1}{\rho_{c}}\left(\frac{d \rho_{\mathrm{GW}}}{d \ln \nu}\right)=\frac{\nu}{\rho_{c}}\left(\frac{d \rho_{\mathrm{GW}}}{d \nu}\right)=\frac{8}{9}\left(\frac{\rho_{\mathrm{ds}}}{\rho_{\mathrm{Planck}}}\right) .
$$

This simple model does not consider the matter dominated era [42]. If we include matter, we have to take into account the equivalence epoch [43]

$$
\Omega_{\mathrm{GW}}^{A}(f)=\frac{8}{9}\left(\frac{\rho_{\mathrm{ds}}}{\rho_{\mathrm{Planck}}}\right)\left(1+z_{e q}\right)^{-1}
$$

where $z_{e q}$ is the redshift at equivalence. This means that the range of frequencies of interest is $\nu>\left(1+z_{e q}\right)^{1 / 2} H_{0}$. The redshift $z_{e q}$ is a correction with respect to the today Hubble parameter $H_{0}$. At lower frequencies, it is [36, 38, 39]

$$
\Omega_{\mathrm{GW}}(\nu) \propto \nu^{-2} .
$$

Furthermore, it is worth noticing that Eqs. (81) and (82) are not correct at any frequency since they do not explicitly depend on frequency. For gravitational waves with frequencies less than $H_{0}$, the energy density is badly defined because their wavelength is larger than the Hubble size of the universe. Similarly, at high frequencies, there is a maximal frequency above which the spectrum rapidly drops to zero. We are assuming that the phase transition from inflation to radiation is instantaneous. In realistic models, this phenomenon occurs over a given time scale related to a gravitational radiation maximal frequency.

However, $\Omega_{\mathrm{GW}}^{A}$ drops rapidly. The cutoffs defined at low and high frequencies indicate that the total energy density of the stochastic gravitational waves has to be finite. These results have been quantitatively constrained taking into account the PLANCK experiment release. On the other hand, since the spectrum drops down as $\propto \nu^{-2}$ for low frequencies, this means that, at LIGO-VIRGO frequencies, one can get

$$
\Omega_{\mathrm{GW}}^{\mathrm{GR}}(\nu) h_{100}^{2}<2 \times 10^{-6}, \quad \Omega_{\mathrm{GW}}^{S}(\nu) h_{100}^{2}<2.3 \times 10^{-12} .
$$


The corresponding strain at $\approx 100 \mathrm{~Hz}$, for VIRGO and LIGO at maximum sensitivity [45, 46] is an interesting quantity to be derived. For this purpose, one can adopt the characteristic amplitude equation for a given component [27]. It is

$$
h_{A}(\nu) \simeq 8.93 \times 10^{-19}\left(\frac{1 \mathrm{~Hz}}{\nu}\right) \sqrt{h_{100}^{2} \Omega_{\mathrm{GW}}(\nu)} .
$$

Finally, we obtain

$$
h_{G R}(100 \mathrm{~Hz})<1.3 \times 10^{-23}, \quad h_{s}(100 \mathrm{~Hz})<2 \times 1.410^{-26} .
$$

where GR and scalar modes are considered.

Because we have sensitivities of the order $10^{-22}$ for the VIRGO and LIGO interferometers at $\approx 100 \mathrm{~Hz}$, one needs to gain more or less three orders of magnitude. At smaller frequencies the sensitivity of the VIRGO interferometer is of the order of $10^{-21}$ at $\approx 10 \mathrm{~Hz}$ and, for the GR and scalar modes, it is

$$
h_{G R}(100 \mathrm{~Hz})<1.3 \times 10^{-22}, \quad h_{S}(100 \mathrm{~Hz})<1.4 \times 10^{-25} .
$$

These numerical results point out that tensor modes can be clearly distinguished by scalar modes. Furthermore, cosmological models can be characterized by the gravitational wave density parameter.

Other features can be put in evidence by the evolution of gravitons in a cosmological background. For example a phantom universe cannot be realized in scalar-tensor gravity but can be realized in the $f(R)$ gravity. In general, it is difficult to distinguish the phantom universe with $w<-1$ from the quintessence universe $w>-1$, if $w$ is almost -1 .

As discussed after Eq. (70), in the phantom universe realized in the context of $f(R)$ gravity, $\hat{h}_{i j}$ is finite but, in the quintessence phase, $\hat{h}_{i j}$ decreases. This fact, according to the analysis performed above, can have dramatic consequences in the measurements of $h$ and of $\Omega_{\mathrm{GW}}$ and therefore we could distinguish, in principle, the phantom universe from the quintessence universe by the stochastic background of gravitational waves.

In the quintessence universe, realized for $\alpha>1$, even if the expansion is identical for the same $\alpha$, the exponents of $\hat{h}_{i j}$ are different for scalar-tensor and $f(R)$ gravity, as shown in (69) and (70). Then by observing the decreasing of graviton in the long range, one can distinguish between scalar tensor and $f(R)$ gravity.

The paradigm presented here can be adopted also for other models (e.g. Galileon, Gauss-Bonnet, etc.) as soon as one is capable of characterizing the source $\delta T_{\mu \nu}$ and a graviton propagation equation (17). Clearly, such an equation depends on the conformal factor in (8) that characterizes both the source and the model. From a genuine observational viewpoint, a main role is played by the upper limit (21) and the amplitude (28). Both of them are related to the Sachs-Wolfe effect and then to the capability of a fine determination of cosmic microwave background temperature anisotropies.

To conclude, we have shown that amplitudes of tensor gravitons and their evolution strictly depend on the cosmological background. This background can be tuned by a scalar field that specifically select the theory of gravity. Conversely, taking into account that primordial vacuum fluctuations produce stochastic gravitons, beside scalar perturbations, kinematical distortions and so on, the initial amplitude of these gravitons is a function of the given theory of gravity. Finally, the Sachs-Wolfe effect could contribute to select a given gravity theory that can be consistently matched with other observations.

\section{Acknowledgements}

This work is supported in part by the INFN sezione di Napoli, iniziative specifiche TEONGRAV and QGSKY (SC and MDL), by MINECO (Spain) project FIS2013-44881 (SDO) by the CSIC I-LINK1019 Project (SC, SN, and SDO). This work is supported (in part) by MEXT KAKENHI Grant-in-Aid for Scientific Research on Innovative Areas "Cosmic Acceleration" (No. 15H05890) (S.N.). The article is also based upon work from COST action CA15117 (CANTATA), supported by COST (European Cooperation in Science and Technology). M.D.L is supported by ERC Synergy Grant "BlackHoleCam" Imaging the Event Horizon of Black Holes awarded by the ERC in 2013 (Grant No. $610058)$.

[1] B. P. Abbott et al. [LIGO Scientific and Virgo Collaborations], Phys. Rev. Lett. 116 (2016) no.6, 061102 doi:10.1103/PhysRevLett.116.061102 arXiv:1602.03837 [gr-qc]]. 
[2] B. P. Abbott et al. [LIGO Scientific and Virgo Collaborations], Phys. Rev. Lett. 116 (2016) no.24, 241103 doi:10.1103/PhysRevLett.116.241103 arXiv:1606.04855 [gr-qc]].

[3] S. Capozziello and M. De Laurentis, Phys. Rept. 509 (2011) 167 doi:10.1016/j.physrep.2011.09.003 arXiv:1108.6266 [grqc]].

[4] S. Nojiri and S. D. Odintsov, Phys. Rept. 505 (2011) 59 doi:10.1016/j.physrep.2011.04.001 arXiv:1011.0544 [gr-qc]].

[5] P. J. E. Peebles and B. Ratra, Rev. Mod. Phys. 75 (2003) 559 doi:10.1103/RevModPhys.75.559 astro-ph/0207347.

[6] V. Sahni, Lect. Notes Phys. 653 (2004) 141 astro-ph/0403324.

[7] K. Bamba, S. Capozziello, S. Nojiri and S.D. Odintsov, Astrophys. Space Sci. 342 (2012) 155 doi:10.1007/s10509-012-1181-8 arXiv:1205.3421 [gr-qc]].

[8] K. Koyama, Rept. Prog. Phys. 79 (2016) no.4, 046902 doi:10.1088/0034-4885/79/4/046902 arXiv:1504.04623 [astroph.CO]].

[9] T. Padmanabhan, Phys. Rept. 380 (2003) 235 doi:10.1016/S0370-1573(03)00120-0 hep-th/0212290.

[10] S. Nojiri and S. D. Odintsov, eConf C 0602061 (2006) 06 [Int. J. Geom. Meth. Mod. Phys. 4 (2007) 115] doi:10.1142/S0219887807001928 hep-th/0601213.

[11] S. Capozziello and M. Francaviglia, Gen. Rel. Grav. 40 (2008) 357 doi:10.1007/s10714-007-0551-y [arXiv:0706.1146] [astro$\mathrm{ph}]$.

[12] Y.-F. Cai, S. Capozziello, M. De Laurentis, E. N. Saridakis, Rept. Prog. Phys. 79, 106901 (2016). doi: 10.1088/00344885/79/10/106901 [arXiv:1511.07586] [gr-qc] ]

[13] W. Lin and M. Ishak, Phys. Rev. D 94 (2016) no.12, 123011 doi:10.1103/PhysRevD.94.123011 arXiv:1605.03504 [astroph.CO]].

[14] M. De Laurentis, O. Porth, L. Bovard, B. Ahmedov and A. Abdujabbarov, Phys. Rev. D 94 (2016) no.12, 124038 doi:10.1103/PhysRevD.94.124038 [arXiv:1611.05766 [gr-qc]].

[15] S. Capozziello, M. De Laurentis and M. Francaviglia, Astropart. Phys. 29 (2008) 125 doi:10.1016/j.astropartphys.2007.12.001 arXiv:0712.2980 [gr-qc]].

[16] S. Bellucci, S. Capozziello, M. De Laurentis and V. Faraoni, Phys. Rev. D 79 (2009) 104004 doi:10.1103/PhysRevD.79.104004 arXiv:0812.1348 [gr-qc]].

[17] C. Bogdanos, S. Capozziello, M. De Laurentis and S. Nesseris, Astropart. $\quad$ Phys. $34 \quad(2010) \quad 236$ doi:10.1016/j.astropartphys.2010.08.001 arXiv:0911.3094 [gr-qc]].

[18] E.A. Novikov, Mod. Phys. Lett. A 31, no. 15 (2016) 1650092, doi: 10.1142/S0217732316500929.

[19] E. A. Novikov, EJTP 13, no. 35 (2016) 79.

[20] M. Pitkin, S. Reid, S. Rowan and J. Hough, Living Rev. Rel. 14 (2011) 5 doi:10.12942/lrr-2011-5 [arXiv:1102.3355] [astroph.IM]].

[21] R.R. Caldwell, Phys. Lett. B 545 (2002) 23 doi:10.1016/S0370-2693(02)02589-3 astro-ph/9908168.

[22] R.R. Caldwell, M. Kamionkowski and N.N. Weinberg, Phys. Rev. Lett. 91 (2003) 071301 doi:10.1103/PhysRevLett.91.071301 astro-ph/0302506.

[23] M. Higuchi and S. Nojiri, Gen. Rel. Grav. 46 (2014) no.11, 1822 doi:10.1007/s10714-014-1822-z arXiv:1402.1346 [hep-th]].

[24] J. C. Fabris and R. de Sá Ribeiro, Gen. Rel. Grav. 32 (2000) no. 11, 2141 doi: 0001-7701/ 00/ 1100-2141\$18.00/ 0

[25] Y. B. Zeldovich, Mon. Not. Roy. Astron. Soc. 160 (1972) 1P.

[26] T. Chiba, T. L. Smith and A. L. Erickcek, Phys. Rev. D 75 (2007) 124014 doi:10.1103/PhysRevD.75.124014 astro-ph/0611867.

[27] M. Maggiore, Phys. Rept. 331 (2000) 283 doi:10.1016/S0370-1573(99)00102-7 gr-qc/9909001.

[28] R. K. Sachs and A. M. Wolfe, Astrophys. J. 147 (1967) 73 [Gen. Rel. Grav. 39 (2007) 1929]. doi:10.1007/s10714-007-0448-9

[29] A.A. Starobinsky, Sov. Phys. JETP Lett. 34, 438 (1982).

[30] P. A. R. Ade et al. [Planck Collaboration], Astron. Astrophys. 594 (2016) A14 doi:10.1051/0004-6361/201525814 arXiv:1502.01590 [astro-ph.CO]].

[31] C. Corda, EJTP 9, no. 26, 1 (2012), arXiv:1110.1772 1 [gr-qc]].

[32] S. Capozziello, C. Corda and M. F. De Laurentis, Mod. Phys. Lett. A 22, no. 15, 1097 (2007), doi: $10.1142 / \mathrm{S} 0217732307023444$.

[33] S. Nojiri and S. D. Odintsov, Gen. Rel. Grav. 38 (2006) 1285 doi:10.1007/s10714-006-0301-6 hep-th/0506212.

[34] A. J. Farmer and E. S. Phinney, Mon. Not. Roy. Astron. Soc. 346 (2003) 1197 doi:10.1111/j.1365-2966.2003.07176.x astro-ph/0304393.

[35] A. Buonanno, Proceedings of the Les Houches Summer School, Particle Physics and Cosmology: The Fabric of Spacetime, Les Houches, France, 31 Jul - 25 Aug 2006, arXiv:0709.4682 (2007).

[36] B. Allen, Proceedings of the Les Houches School on Astrophysical Sources of Gravitational Waves, eds. Jean-Alain Marck and Jean-Pierre Lasota (Cambridge University Press, Cambridge, England 1998).

[37] B. Allen and A. C. Ottewill, Phys. Rev. D 56 (1997) 545 doi:10.1103/PhysRevD.56.545 gr-qc/9607068.

[38] L. P. Grishchuk, V. M. Lipunov, K. A. Postnov, M. E. Prokhorov and B. S. Sathyaprakash, Phys. Usp. 44 (2001) 1 [Usp. Fiz. Nauk 171 (2001) 3] doi:10.1070/PU2001v044n01ABEH000873 astro-ph/0008481.

[39] S. Capozziello, R. Cianci, M. De Laurentis, S. Vignolo Eur. Phys. J. C 70, 341 (2010).

[40] C. Corda Gen. Rel. Grav. 42, 1323 (2010); doi:10.1007/s10714-009-0895-6 10.1007/s10714-009-0917-4 arXiv:0909.4133 [gr-qc]

[41] C. Corda, Eur. Phys. J. C 65, 257 (2010). doi:10.1140/epjc/s10052-009-1100-5 arXiv:1007.4077] [gr-qc]

[42] S. Capozziello, M. De Laurentis, Int. J.Mod. Phys. Conf. Ser 14, 260 (2012)

[43] B. Allen, Phys. Rev. D 37 (1988) 2078. doi:10.1103/PhysRevD.37.2078 
[44] S. Capozziello, M. De Laurentis, S. Nojiri and S. D. Odintsov, Gen. Rel. Grav. 41 (2009) 2313 doi:10.1007/s10714-0090758-1 arXiv:0808.1335 [hep-th]].

[45] http://www.ligo.org/pdf public/camp.pdf.

[46] http://www.virgo.infn.it 